\title{
Factores de riesgo de carga en cuidadores informales de adultos mayores con demencia
}

\author{
Risk factors of burden in informal caregivers of older adults \\ with dementia
}

\author{
Dra. C. Ana Margarita Espín Andrade \\ Instituto superior de Ciencias Médicas de La Habana. La Habana, Cuba.
}

\section{RESUMEN}

Introducción: el fenómeno del envejecimiento de la población cubana es hoy una realidad que impone grandes retos a toda la sociedad y en particular a la familia, principal fuente de protección y apoyo para sus personas mayores.

Objetivos: diagnosticar la carga e identificar sus factores de riesgo en los cuidadores informales de adultos mayores con demencia.

Métodos: investigación analítica, de corte transversal, realizada en 192 cuidadores que acudieron a las consultas de los Equipos Multidisciplinarios de Atención Geriátrica del Centro I beroamericano para la Tercera Edad y de áreas de salud de los municipios Playa y Plaza de la Revolución, de La Habana, durante el período 2003-2009. Se estudiaron variables del paciente, del cuidador y de la familia. La información se obtuvo a partir de la aplicación de diferentes instrumentos, entre ellos, cuestionario de caracterización del cuidador, escala de carga de Zarit y prueba de funcionamiento familiar. Para identificar los factores de riesgo de carga, se diseñó un estudio de casos y controles y el procedimiento estadístico empleado fue la regresión logística.

Resultados: los cuidadores eran mayormente hijas de edad mediana con afectaciones de salud, elevado consumo de fármacos y limitaciones económicas; no tenían experiencia previa y no contaban con conocimientos acerca de la enfermedad y su manejo.

Conclusiones: la carga se identifica en la mayoría de los cuidadores y sus factores de riesgo son, entre otros, grado de dependencia del enfermo, depresión del cuidador y el funcionamiento familiar. La edad del cuidador es ser un factor protector de carga.

Palabra clave: cuidador informal, adulto mayor dependiente, demencia, carga, factores de riesgo. 


\section{ABSTRACT}

Introduction: aging of the Cuban population is considered to be an achievement; however, it is a present reality that brings big challenges to the whole society, particularly the family which is the main source of protection and support for elders. Objectives: to diagnose the burden and to identify its risk factors in the informal caregivers of old people with dementia.

Methods: cross-sectional analytical research conducted in 192 caregivers, who went to the consultation service of the multidisciplinary geriatric care teams in the I bero-American Institute for the Old Age and in the health areas located in Playa and Plaza municipalities from 2003 to 2009. The variables related to the patient, the caregiver and the family were studied. The collected information stemmed from the administration of different instruments such as caregiver characterization questionnaire, Zarit's burden scale and the family functioning test. A case-control study was designed and the logistic regression technique was used in order to identify the risk factors of burden.

Results: the caregivers were mostly middle-aged daughters having health problems, high consumption of pharmaceuticals and economic restrictions. They were generally inexperienced and lacked appropriate knowledge about dementia and how to manage it.

Conclusions: the burden is present in most of the caregivers and the main risk factors are degree of patient's dependence on the caregiver, depression and family functioning. The age of the caregiver is the protecting factor in terms of burden.

Key words: informal caregiver, dependent aged person, dementia, burden, risk factors.

\section{NTRODUCCI ÓN}

El envejecimiento de la población en Cuba es similar al de muchos países desarrollados, y se encuentra entre los que tienen un índice más elevado dentro del grupo de países en desarrollo. Esto es el resultado de la disminución de la fecundidad, el aumento de la esperanza de vida al nacer y la disminución de la mortalidad del grupo de adultos mayores, que ha sido favorecido por la existencia de un sistema de salud accesible, que jerarquiza la atención a los grupos más vulnerables de la población, para lo cual cuenta con un Programa Nacional de Atención al Adulto Mayor. ${ }^{1}$ Este fenómeno, si bien se considera un logro, es hoy una realidad que impone grandes retos a toda la sociedad, ${ }^{2}$ y en particular a la familia, la que sigue siendo la principal fuente de protección y apoyo para sus personas mayores.

La familia en su conjunto sufre un cambio en su dinámica y funcionamiento cuando uno de sus miembros envejecidos presenta alguna enfermedad, sobre todo si hay una pérdida de autonomía del anciano para llevar a cabo sus actividades de la vida diaria, y necesita del apoyo de otros para realizarlas y satisfacer sus necesidades. Generalmente, este deterioro funcional provoca alteraciones emocionales y cognitivas que agravan la situación del anciano, aumenta la tensión de los miembros de la familia, y se hace más difícil la tarea del cuidado para quienes asumen esta responsabilidad dentro de ella. 
Una de las enfermedades que más deterioro funcional y por tanto, mayor estrés proporcionan al cuidador, es la demencia. Esta es una enfermedad del sistema nervioso central, dada por alteraciones de los procesos mentales superiores, que modifica la personalidad y la conducta de las personas que la padecen. Si bien algunos tipos de demencia pueden presentarse antes de los 60 años, la gran mayoría de ellas aparecen después de esta edad y se duplica por cada década de la vida en el caso de algunas, como por ejemplo, en la enfermedad de Alzheimer, que se considera el tipo más frecuente. ${ }^{3}$ Esto quiere decir que, a medida que se incrementa el índice de envejecimiento, aumenta el riesgo de padecer esta enfermedad, la cual tiene un elevado costo a nivel individual, familiar y social.

Se estima por estudios realizados en algunas localidades del país, que la prevalencia de la demencia en Cuba es alta, y en particular de la enfermedad de Alzheimer. ${ }^{4}$

Los cuidadores de adultos mayores que sufren demencia se afectan en el orden físico, psíquico y socioeconómico, lo que conlleva a un elevado estrés que repercute en la calidad del cuidado que recibe el adulto mayor y en la propia evolución de la enfermedad o discapacidad. La aparición de afecciones óseas, cardiovasculares y otras de índole físico; la aparición de trastornos emocionales como depresión y ansiedad así como la alteración de sus relaciones laborales y sociales, con la consecuente merma de sus recursos económicos; son algunos ejemplos de las consecuencias negativas del cuidado mantenido de una persona con esta enfermedad. ${ }^{5}$

La afectación que experimenta el cuidador y el sentimiento de que su vida ha cambiado desfavorablemente a partir del cuidado del anciano, es resumida en el concepto de "carga". La carga se ha convertido en un predictor de la calidad del cuidado que ofrece el familiar y también de la solicitud de institucionalización por parte de la familia del anciano que sufre demencia. ${ }^{6}$ Resulta necesario prevenir la carga, para lo que es imprescindible identificar los factores de riesgo que inciden en su aparición. Las variables que influyen en la carga del cuidador son múltiples y muy heterogéneas, pues pueden estar relacionadas con el propio enfermo, con las características del cuidador, o referidas a la relación anterior y actual entre los miembros de la familia.

Si bien se reconoce la implicación que tiene este problema de salud, no solo para el paciente sino para el cuidador, la familia y la sociedad en general, es frecuente que en la práctica cotidiana, los profesionales de la salud dirijan su atención y recursos al manejo de las enfermedades del paciente, sin tener en cuenta el contexto familiar donde se genera un sinnúmero de cambios. Es necesario atender al binomio paciente-cuidador, como parte del tratamiento a la enfermedad.

El presente trabajo tiene el propósito de diagnosticar la presencia de carga en los cuidadores informales e identificar los factores de riesgo que conducen a esta, lo que permite en lo adelante trazar estrategias de intervención para mejorar la calidad de vida de enfermos y cuidadores.

\section{MÉTODOS}

Se realizó un estudio analítico de corte transversal en 192 cuidadores informales de adultos mayores con demencia, que constituyó la totalidad de cuidadores que acudieron a las consultas de Psicología planificadas para los efectos de la investigación, previa remisión y diagnóstico de demencia a los pacientes, por parte 
de los Equipos Multidisciplinarios de Atención Geriátrica (EMAG) de cada uno de los centros seleccionados (Centro de Estudios de Longevidad, Envejecimiento y Salud (CITED), áreas de salud de los municipios Playa y Plaza de la Revolución), durante los años comprendidos entre 2003-2009.

\section{Criterios de inclusión}

- Cuidadores de adultos mayores con diagnóstico de demencia.

- Cuidadores con voluntad de participar en el estudio.

\section{Criterios de exclusión}

- Cuidadores de 60 años o más, con deterioro cognitivo

Las variables independientes fueron las del paciente, del cuidador y de la familia.

\section{Instrumentos para obtener la información acerca de las variables del cuidador}

1. Cuestionario de caracterización del cuidador. Creado y validado por la autora del presente trabajo, consta de 19 preguntas cerradas que recogen datos sociodemográficos y otras características del cuidador relacionadas con la atención al enfermo. ${ }^{7}$

2. Escala de carga de Zarit. Fue construida en 1983 por Zarit y Zarit, consta de 21 ítems y mide tres dimensiones subyacentes a la carga: impacto de cuidado, carga interpersonal y expectativas de autoeficacia. ${ }^{8}$

3. Escala de Depresión, de Beck. Uno de los instrumentos más utilizados para evaluar la existencia o severidad de síntomas de depresión, dada su probada validez y confiabilidad tanto en poblaciones clínicas como no clínicas. La prueba, que consta de 21 ítems, tiene como objetivo identificar síntomas típicos de la depresión. ${ }^{9}$

\section{Instrumentos para obtener la información sobre el adulto mayor con demencia}

1. Clinical Dementia Rating Scale (CDR). Valora el momento evolutivo sobre la base del rendimiento del sujeto en seis modalidades de tipo cognitivo y funcional. Las modalidades evaluadas son: memoria, orientación, razonamiento, actividades sociolaborales, actividades recreativas, y cuidado personal. La escala establece cinco posibles estadios desde la normalidad, demencia cuestionable, demencia leve, demencia moderada y demencia severa. La afectación del área "memoria" tiene primacía para determinar el estadio general. ${ }^{10}$

2. Escala de Katz. Una de las pruebas más conocida, estudiada y validada.

Elaborado en 1958 por un grupo multidisciplinario del hospital "Benjamín Rose" de Cleveland para enfermos hospitalizados con fractura de cadera. Evalúa las actividades básicas de la vida diaria (AIVD). Consta de 6 ítems: baño, vestido, uso del retrete, movilidad, continencia, alimentación. ${ }^{11}$

3. Escala de Lawton y Brody. Instrumento publicado en 1969 y construido para población anciana. Recoge información sobre las actividades instrumentales de la 
vida diaria (AIVD) recogidas en 8 ítems: usar el teléfono, ir de compras, preparar la comida, realizar tareas del hogar, lavar la ropa, utilizar transporte, controlar la medicación, manejar el dinero. Es predictivo de las actividades básicas durante un ingreso hospitalario, por lo que algunos autores han sugerido que puede ser un indicador de fragilidad. ${ }^{12}$

4. Inventario Neuropsiquiátrico (NPI). La versión en español es un instrumento fiable para valorar de una forma breve los síntomas no cognitivos en pacientes con demencia. Es un instrumento que ha demostrado ser útil tanto para la investigación, como para la práctica clínica en diferentes culturas de todo el mundo. ${ }^{13}$

\section{Instrumento para obtener la información sobre la familia}

1. Prueba de Funcionamiento Familiar FF-SIL. Diseñado en Cuba, con el objetivo de evaluar el funcionamiento familiar, considerado como la dinámica relacional sistémica que se da entre los miembros de la familia. Consta de 14 proposiciones y 7 categorías que definen el funcionamiento familiar. ${ }^{14}$

Para estudiar las relaciones existentes entre las variables seleccionadas y la presencia o no de carga en los cuidadores, se confeccionaron tablas de contingencia, y se aplicó la prueba de independencia ji-cuadrado de Pearson, para detectar asociación significativa. Se consideró como nivel de significación de la prueba un $\alpha=0,05$.

Para profundizar en la relación de dependencia entre la carga del cuidador y un grupo de variables independientes, es decir, para identificar los factores de riesgo de carga, se diseñó un estudio de casos y controles. Las variables independientes que se estudiaron fueron trastornos de conducta y grado de dependencia del paciente, y edad del cuidador, por haber sido estadísticamente significativas en el análisis univariado inicial. Se incluyeron además otras variables del cuidador como: sexo, convivencia, tiempo de cuidador, parentesco, apoyo recibido, situación económica, estado de salud, estrategia de afrontamiento, información acerca de la enfermedad y depresión, así como la variable funcionamiento familiar. Se seleccionaron todas ellas por ser las que más se han relacionado con la carga en estudios revisados. ${ }^{15-17}$

El procedimiento estadístico que se empleó fue la regresión logística. Para probar la bondad de ajuste del modelo, se utilizó la prueba estadística Hosmer y Lemeshow.

\section{RESULTADOS}

El 52,1 \% de cuidadores tenía carga y 47,9 \% no presentó carga.

En la aplicación de la regresión logística para identificar los factores de riesgo que conducen a la carga, se comprobó que hubo ajuste al modelo, donde el estadígrafo Hosmer y Lemeshow presentó una $p=0,656$.

Los valores del nivel de significación menores de 0,05 indicaron que los factores de riesgo de la carga fueron: la edad joven del cuidador $(0,02)$, la dependencia funcional del enfermo $(0,01)$, el padecimiento de enfermedades del cuidador $(0,00)$, la disfunción familiar $(0,00)$ y la depresión del cuidador $(0,00)$. Los 
trastornos de conducta del enfermo, aunque obtuvieron un $\alpha=0,05$, fue considerado también un factor de riesgo (tabla).

Tabla. Factores de riesgo de carga del cuidador

\begin{tabular}{|c|c|c|c|c|c|}
\hline \multirow[t]{2}{*}{ Variables } & \multirow[t]{2}{*}{$\beta$} & \multirow[t]{2}{*}{ Sig. } & \multirow[t]{2}{*}{$\operatorname{Exp} \cdot(\beta)$} & \multicolumn{2}{|c|}{$\begin{array}{c}\text { I.C. } 95 \% \text { para } \\
\text { Exp. }(\beta)\end{array}$} \\
\hline & & & & Inferior & Superior \\
\hline Edad del cuidador & $-0,784$ & 0,026 & 0,457 & 0,229 & 0,910 \\
\hline Estado funcional del enfermo & 0,816 & 0,019 & 2,261 & 1,142 & 4,475 \\
\hline Estado de salud del cuidador & 1,150 & 0,009 & 3,157 & 1,328 & 7,506 \\
\hline Funcionamiento familiar & 2,602 & 0,000 & 13,484 & 4,511 & 40,308 \\
\hline Depresión del cuidador & 2,935 & 0,000 & 18,829 & 6,964 & 50,908 \\
\hline $\begin{array}{l}\text { Trastorno de conducta } \\
\text { del enfermo }\end{array}$ & 0,612 & 0,055 & 1,844 & 0,988 & 3,442 \\
\hline
\end{tabular}

La edad del cuidador resultó ser un factor de protección lo que se apreció en el valor negativo de $\beta(-0,784)$, lo que indicó que a medida que aumentó la edad, disminuyó la carga.

Cuando el paciente es dependiente, es 2,26 veces más probable que el cuidador presente carga, no es así cuando es dependiente parcial, y en la misma medida, cuando es dependiente parcial a cuando es independiente. Quiere decir que a medida que aumenta la dependencia del paciente, es mayor la carga.

También es 3,15 veces más probable que el cuidador tenga carga cuando padece enfermedades, que cuando no las padece. Por otra parte, es 13,48 más probable que el cuidador tenga carga cuando la familia es disfuncional que cuando no lo es.

En relación con la depresión, es 18,89 veces más la probabilidad de que el cuidador tenga carga cuando tiene depresión que cuando no la tiene. Es 1,84 más probable que el cuidador tenga carga cuando el paciente presenta trastorno de conducta que cuando no lo presenta.

\section{DISCUSIÓN}

A pesar de que el número de cuidadores con carga es alto, se pudiera pensar en un subregistro, debido a que los cuidadores muchas veces ocultan el daño que les hace el cuidado de un familiar, confesar que experimentan carga les puede ocasionar sentimiento de culpa, lo cual puede incidir en que no sean totalmente sinceros en las respuestas al cuestionario. Se ha encontrado que el cuidador no suele comentar al médico los problemas que padece, y solo lo hace cuando ya son graves. Otro estudio que también utilizó la escala de Zarit encontró que la carga se presentó en el $47 \%$ de los cuidadores. ${ }^{18}$

Por otra parte, se ha visto que el cuidado de un familiar que sufre demencia no siempre tiene consecuencias negativas para el cuidador, y en este aspecto se ha señalado: 
la mayoría de las cuidadoras, después de un tiempo de estar al cuidado de un anciano enfermo, y luego de hacer los ajustes necesarios en el estilo de vida, asumen el rol de cuidado con satisfacción y humildad; algunas abrigan la esperanza de un reconocimiento futuro cuando serán premiadas por la dedicación a esa persona a quien le han dado sus mejores cuidados. ${ }^{19}$

El hecho de que a medida que aumentó la edad, disminuyó la carga pudiera explicarse porque los cuidadores mayores tienen menos roles de otro tipo que cumplir, así como tienen menos responsabilidades laborales y sociales, como señala el estudio realizado en España, en el año 2004, por Mahoney y otros. ${ }^{20}$ Este resultado coincide con el de otro estudio que encuentra que el cuidador sobrecargado es el más joven, es decir, que a menor edad, más carga, lo que pudiera explicarse por el hecho de que la persona joven recién comienza su vida laboral, y la asunción de un nuevo rol que debe armonizar con otras tareas, supone una fuente incesante de estrés, por lo que la carga es mayor. ${ }^{21}$

La relación entre la dependencia del enfermo y la carga ha sido hallada en otros estudios. ${ }^{22,23}$ Esto es un resultado esperado, ya que a medida que el paciente tiene menos validismo, el cuidador tiene que realizar más tareas. Por otra parte, la imagen acerca de la persona cuidada, que la mayor parte de las veces es un familiar muy allegado, se desmorona por la pérdida de capacidades y de autonomía del enfermo, lo que provoca sentimientos y emociones negativos que pueden repercutir en la carga.

Otro estudio también encuentra que el mayor grado de carga estaba relacionado de forma significativa con alteraciones físicas (molestias musculares) y mentales (ansiedad) en el cuidador principal. ${ }^{24}$

Himes y Reidy hallan relación entre el funcionamiento familiar y la carga, y plantean que una adecuada función familiar puede asegurar por un lado, que el anciano continúe viviendo en la comunidad y por otro, que el cuidador no desarrolle sobrecarga para el cuidado ni tenga repercusiones negativas en su estado de salud física, mental y social. ${ }^{25}$

Un estudio realizado en Colombia en el 2006 apunta que la disfunción familiar severa es más frecuente en los cuidadores con carga. ${ }^{18}$

La depresión es una de las alteraciones psíquicas que más afecta a los cuidadores desde el comienzo de la enfermedad, en los que va a existir un duelo anticipado, debido a que asisten al deterioro de las funciones mentales y físicas de su familiar. Se unen otros factores como el escaso tiempo de que disponen, el abandono de sus propias necesidades, la no comprensión de que de su propio cuidado depende la evolución del enfermo, y otros, lo que empeora la atención a este problema tan frecuente en ellos. Lo anterior provoca que no asistan a los servicios de salud a solicitar ayuda, lo que conduce a la cronicidad de la depresión y a la afectación del sistema inmune, aparecen o se descompensan las enfermedades crónicas y se agrava la crisis del sistema familiar.

El trastorno de conducta del enfermo es una de las variables que más se ha estudiado en relación con la carga, ya que a los cuidadores les resulta extremadamente difícil y angustiosa la presencia de problemas con los cuales antes no había lidiado, máxime cuando es un familiar allegado quien los presenta. Se ha observado que la presencia de trastornos del comportamiento se asocia significativamente de forma inversa con la salud y el bienestar del cuidador. ${ }^{26}$ 
El problema personal, psíquico y social de los cuidadores de este estudio coincidió con la de los cuidadores de otras latitudes. Sin embargo, se demostró que no todas las variables del enfermo y del cuidador que se plantean en la literatura, incidieron en la carga de los cuidadores, sino sólo algunas de ellas constituyeron factores de riesgo de la misma, lo que es importante tomar en cuenta en el diseño de intervenciones psicoeducativas.

\section{REFERENCI AS BI BLI OGRÁFICAS}

1. Torres Vidal RM, Gran Álvarez MA. Panorama de la salud del adulto mayor en Cuba. Rev Cubana Salud Pública [Internet]. 2005 [citado 23 Mar 2012]; 31. Disponible en: http://bvs.sld.cu/revistas/spu/vol31_2_05/sp06205.htm

2. Rodríguez Cabrera A, Álvarez Vázquez L. Repercusiones del envejecimiento de la población cubana en el sector salud. Rev Cubana Salud Pública [Internet]. 2006 [citado 23 Mar 2012]; 32(2). Disponible en:

http://scielo.sld.cu/scielo.php?script=sci arttext\&pid=S086434662006000200013\&lng=es\&nrm=iso\&ting=es

3. Llibre Guerra JC, Guerra Hernández M, Perera Miniet, E. Comportamiento del síndrome demencial y la enfermedad de Alzheimer. Rev Haban Cienc

Méd. 2008; 7(1). Disponible en:

http://scielo. sld.cu/scielo.php?script=sci arttext\&pid=S1729-

$\underline{519 \times 2008000100008 \& \operatorname{lng}=e s \& n r m=i s o / \& \ln g=e s}$

4. _._. Comportamiento de las enfermedades crónicas no transmisibles en adultos mayores. Rev Cubana Med Gen Integr [Internet]. 2008 [citado 23 Mar 2012];24(4). Disponible en:

http://scielo.sld.cu/scielo.php?script=sci arttext\&pid=S0864-

$\underline{21252008000400005 \& \operatorname{lng}=e s \& n r m=i s o \& t \operatorname{lng}=e s}$

5. Espín AM. Caracterización psicosocial de cuidadores informales de adultos mayores con demencia. Rev Cubana Salud Pública [Internet]. 2008 [citado 23 Mar 2012];34(3). Disponible en:

http://scielo.sld.cu/scielo.php?script=sci arttext\&pid=S0864-

$\underline{34662008000300008 \& \operatorname{lng}=e s \& n r m=i s o \& \operatorname{ting}=e s}$

6. Espín AM, Picardi P. Cuidado del adulto mayor dependiente: Impacto en un grupo de cuidadores mexicanos. Revista Tiempo. 2007;21. Disponible en:

http://www.psicomundo.com/tiempo/Tiempo21.pdf

7. Espín AM, Seco T. Metodología de intervención educativo-terapéutica en cuidadores de ancianos con demencia de Alzheimer. Rev Arg Geriatr. 1998; 18:18-27.

8. Montorio I, Fernández MI, López A, Sánchez M. La entrevista de carga del cuidador. Utilidad y validez del concepto de carga. Anal psicol. 1998;14(2):229-48.

9. Beck A, Steer R, Brown G. Inventario de depresión de Beck. 2da ed. Argentina: Editorial Paidós; 2004.

http://scielo.sld.cu 
10. Hughes CP, Berg L, Danzinger WL, Coben LA, Martín RL. A new clinical scale for the staging of dementia. Br J Psychiatry. 1982;140:566-72.

11. Katz S, Ford AB, Moskowitz RW, Jakson BA, Jaffe MW. Studies of in the aged. The index of ADL: a standardized measure of biological and psychological function. J AMA. 1963; 185:914-9.

12. Lawton MP, Brody EM. Assessment of older people: self maintaining and instrumental activities of daily living. Gerontologist. 1969;9:179-86.

13. Vilalta J, Lozano M, Llinás R, López S. Neuropsychiatric inventory. Propiedades psicométricas de su adaptación al español. Rev Neurol. 1999;29(1).

14. Ortíz MT, Louro I, J iménez L, Silva LC. Proyectos de Intervención en Salud Familiar: una propuesta método. Rev Cubana Salud Pública [Internet]. 2000 [citado 23 Mar 2012];26(1): 12-6. Disponible en: http://scielo.sld.cu/scielo.php?script=sci arttext\&pid=S0864$\underline{34662000000100002 \& \operatorname{lng}=\mathrm{es} \& \mathrm{nrm}=\mathrm{iso} \& \mathrm{t} \operatorname{lng}=\mathrm{es}}$

15. Steve F, Pedraza V. Demencia, sobrecarga y extrañamiento: Programa de asistencia familiar. Umbral Científico. 2006; 7:96-110.

16. Llibre JJ, Prince M. Demencias y enfermedad de Alzheimer en la población cubana. Impacto de las demencias en la familia. Estudio 10/66 en Ciudad de la Habana y Matanzas. La Habana: Editorial Científica-Técnica; 2008.

17. de la Cuesta C, Sandelowsky M. Tenerlos en casa. The material World and craft of caregiving for relatives with dementia. Transcul Nursing. 2005; 16(3):218-25.

18. Dueñas E, Martínez MA, Morales B, Muñoz C, Viáfara, AS, Herrera JA. Síndrome del cuidador de adultos mayores discapacitados y sus implicaciones psicosociales. Colomb Med. 2006;37(2) supl. 1:1-7.

19. López J, Crespo M. Intervenciones con cuidadores de familiares mayores dependientes: una revisión. Psicothema. 2007;19(1):72-80.

20. Loukissa DA. Family burden in chronic mental illness: a review of research studies. J Adv Nurs. 1995;21:248-55.

21. Molina Linde J M, Láñez Velasco MA. El apoyo social como modulador de la carga del cuidador de enfermos de Alzheimer. Psicología y salud. 2005; 15(1):33-43.

22. Farran CJ, Keane Hagerty E, Tatrowicz L, Scorza E. Dementia care-receiver needs and their impact on caregivers. Clin Nurs Res. 1993;2:86-97.

23. Pearson J, Verma S, Nellet C. Elderly psychiatric patient status and caregiver perception as predictor of caregiver burden. Gerontologist. 1988;28:79-83. 
24. Alonso A, Garrido A, Díaz R, Casquero R, Riera M. Perfil y sobrecarga de los cuidadores de pacientes con demencia incluidos en el programa ALOIS. Atención Primaria. 2004;33(2):67-80.

25. Himes $\mathrm{CL}$, Reidy EB. The role of friends in caregiving. Res Aging. 2006; 22(4): 315-36.

26. Roca AC, Blanco K. Carga en familiares cuidadores de ancianos dementes. Correo Científico Médico Holguín. 2007.11(4): supl 1.

Recibido: 11 de abril de 2011.

Aprobado: 8 de febrero de 2012.

Ana Margarita Espín Andrade. Instituto Superior de Ciencias Médicas de la Habana. Calle 52 No. 4120 e/41 y 43 Playa. La Habana, Cuba. Telf: 209-5966. Correo electrónico: amespin@infomed.sld.cu 